\title{
La relación entre el desempeño y el aprendizaje en las organizaciones
}

\author{
María del Carmen Gómez Romo \\ Universidad Técnica de Ambato \\ Ecuador \\ Telmo Diego Proaño Córdova \\ Universidad Técnica de Ambato \\ Ecuador \\ Andrés Francisco López Gómez \\ Universidad Técnica de Ambato \\ Ecuador
}




\title{
La relación entre el desempeño y el aprendizaje en las organizaciones
}

\author{
María del Carmen Gómez Romo ${ }^{1}$ (D) Telmo Diego Proaño Córdova ${ }^{1}$ y Andrés \\ Francisco López Gómez ${ }^{1}$ (D) \\ 1 Universidad Técnica de Ambato - Ecuador
}

\section{RESUMEN}

El aprendizaje es una herramienta para el desarrollo y sostenibilidad de las organizaciones que propicia un desempeño eficiente, y permite que estas se conviertan en organizaciones inteligentes. El objetivo de la presente investigación fue determinar la relación que existe entre el desempeño y el aprendizaje en la Facultad de Contabilidad y Auditoría en una universidad pública en la zona centro del Ecuador. Las organizaciones enfrentan obstáculos en varias fases de la transferencia del conocimiento, desde lo individual hasta lo colectivo, obstáculos que pueden ser superados a través del aprendizaje. Se investigó los fundamentos teóricos que respaldan el estudio y se aplicó una encuesta estructurada con 23 items (5 de adquisición del conocimiento, 5 de distribución del conocimiento, 4 de memoria organizacional, 4 de desaprendizaje y 5 desempeño) a una muestra aleatoria de 63 docentes de un universo de 75 . El cuestionario fue validado con un alfa de Cronbach 0,918. El procesamiento de datos permitió determinar los estadísticos descriptivos de las dimensiones del aprendizaje y del desempeño. Se encontró evidencia estadística significativa de que existe una muy buena correlación positiva entre el aprendizaje y el desempeño, a un nivel de significancia del $5 \%$ y que el $76,2 \%$ del desempeño fue explicado por las dimensiones del aprendizaje organizacional. Por otro lado, al aplicar una lista de chequeo al plan estratégico de la facultad, no se encontraron elementos del aprendizaje y desempeño.

Palabras clave: aprendizaje organizacional; evaluación del desempeño; facultad de contabilidad y auditoria; gestión organizacional; sostenibilidad.

JEL: M12

\section{The relationship between performance and learning in organizations}

\begin{abstract}
Learning is a tool for the development and sustainability of organizations that promotes efficient performance, and allows them to become intelligent organizations. The objective of this research was to determine the relationship between performance and learning in the School of Accounting and Auditing of a public university, in the central area of Ecuador. Organizations face obstacles in various phases of knowledge transfer, from individual to collective, but these obstacles can be overcome through learning. The theoretical bases relevant to the study were examined and a structured survey with 23 items (5 for knowledge acquisition, 5 for knowledge distribution, 4 for organizational memory, 4 for unlearning and 5 for performance) was applied to a random sample of 63 teachers out of a universe of 75 . The questionnaire was validated with a Cronbach's alpha 0.918. The data processing allowed to determine the descriptive statistics of the learning and performance dimensions. Significant statistical evidence was found that there is a very good positive correlation between learning and performance, at a significance level of $5 \%$ and that $76.2 \%$ of performance was explained by the dimensions of organizational learning. On the other hand, when applying a checklist to the strategic plan of the school, no elements of learning and performance were found.
\end{abstract}

Keywords: organizational learning; performance evaluation; school of accounting and auditings; organizational management; sustainability.

JEL: M12

Copyright: (C) 2020. Gómez, M., Proaño, T., y López, A. Este es un artículo de acceso abierto, https://creativecommons.org/licenses/by-nc-

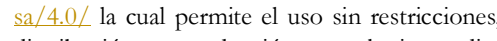
distribución y reproducción en cualquier medio, cuando que el original, el autor y la

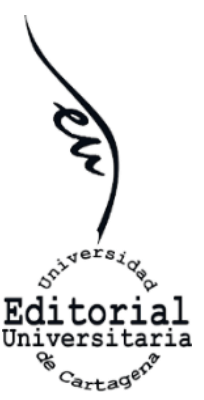




\section{INTRODUCCIÓN}

Todas las personas deben desarrollar nuevas competencias en las organizaciones, o por lo menos, buscar el perfeccionamiento de aquellas adquiridas, a fin de mejorar constantemente su desempeño en el puesto de trabajo (Alles, 2005); esto se logra mediante el aprendizaje. Un buen sistema de Gestión del Talento Humano debe ser capaz de integrar, de principio a fin, con objetividad todos los procesos relacionados con las personas en los que se asocian de manera eficiente los recursos a los objetivos organizacionales. Dentro de la Gestión del Talento Humano, en la categoría de desarrollo personal y profesional, se enfatiza la necesidad del desarrollo de competencias y aprendizaje (Sanabria, Telch, Rodas, Astudillo, \& Estrada, 2015), por tanto, es algo en lo que se debe invertir tiempo y esfuerzo, dado que en toda organización, se desarrollan múltiples aprendizajes que son trasladados, del plano personal al plano organizacional.

El proceso de transferencia de conocimiento de lo individual a lo colectivo, enfrenta un sin número de obstáculos que pueden ser superados con el aprendizaje organizacional. El aprendizaje es sin duda una herramienta de supervivencia y desarrollo de las organizaciones que permite que estas se conviertan en organizaciones inteligentes (Gómez - Romero, Villareal D. \& Villareal F., 2015). La transferencia del conocimiento, ocurre en todo tipo de organizaciones, sin que dependa de su naturaleza, sin embargo, su presencia se hace más visible en las instituciones educativas en virtud de que su desenvolvimiento gira alrededor de éste. En Ecuador, en la búsqueda del mejoramiento de la calidad educativa, las instituciones de educación superior (IES), que incluyen a las Universidades y Escuelas Politécnicas, están sometidas periódicamente a evaluación por parte del Consejo de Evaluación, Acreditación y Aseguramiento de la Calidad de la Educación Superior (CEAACES), por tanto, existe un estrecho monitoreo enfocado a la mejora continua que se evidencia en los aprendizajes.

EI CEAACES dispone el Modelo oficial de Evaluación Institucional de Universidades y Escuelas Politécnicas, bajo la cobertura de la Ley Orgánica de Educación Suprior (LOES) que manifiesta que la evaluación: Es el proceso de verificación que el Consejo de Evaluación, Acreditación y Aseguramiento de la Calidad de la Educación Superior realiza a través de pares académicos de la totalidad o de las actividades institucionales o de una carrera o programa para determinar que su desempeño cumple con las características y estándares de calidad de las instituciones de educación superior y que sus actividades se realizan en concordancia con la misión, visión, propósitos y objetivos institucionales o de carrera, de tal manera que pueda certificar ante la sociedad la calidad académica y la integridad institucional (LOES, 2010, art. 100). Por lo indicado, las IES deben asumir el reto que implica conseguir la acreditación institucional, mostrando evidencias de su desempeño en distintos ámbitos como organización, academia, investigación, vinculación con la sociedad, recursos e infraestructura y estudiantes (CEAACES, 2015). Es en este escenario, que en la Facultad de Contabilidad y Auditoría de la Universidad Técnica de Ambato (UTA), se llevó a cabo esta investigación referente al aprendizaje organizacional con miras a instituirlo de manera sostenida dentro de cada uno de sus procesos internos.

Según Castañeda \& Ríos (2007), el aprendizaje organizacional es el proceso a través del cual las personas adquieren, construyen y transfieren conocimiento. Este proceso ocurre 
en todos los niveles de la organización y dota a ésta de una adecuada capacidad de reacción para adaptarse y responder a cambios del entorno (Aramburu, 2000); condición sin la cual, se habrían levantado barreras que impidan avanzar (Senge, 2012). Más allá de la adaptación al entorno y cumplimiento de estándares, las organizaciones deben incrementar sus capacidades y entrar en nuevas etapas de crecimiento y de autotransformación. La perspectiva de aprendizaje y crecimiento es abordada desde la Gestión de Talento Humano, y amerita quizá la mayor atención y esfuerzo posible, en virtud del alto impacto que tiene en las organizaciones (Del Río Cortina \& Rojas, 2011), así mismo, el desarrollo personal y el desempeño, juegan un papel preponderante en el desenvolvimiento organizacional (Sanabria et al., 2015), dado que, son el motor de las organizaciones. Todo desempeño debe ser evaluado, y su evaluación consiste en el monitoreo y medición de los resultados obtenidos, en función de aquellos propuestos, con lo que se determina su impacto en el cumplimiento de los objetivos organizacionales. A la evaluación del desempeño, en algunas ocasiones se la conoce como auditoría de recursos humanos (Chiavenato, 2007) y es un punto crítico, a partir del cual se definen nuevas estrategias para el efectivo cumplimiento de la misión en las organizaciones.

El objetivo de la presente investigación es determinar la relación que existe entre el desempeño y el aprendizaje organizacional, a partir de los datos provenientes de las encuestas aplicadas a los docentes de la Facultad de Contabilidad y Auditoría (FCAUD). Los resultados obtenidos en esta investigación abren perspectivas para la inclusión de aspectos del aprendizaje en el plan estratégico de la FCAUD, que contribuyan en la generación de evidencias de un desempeño eficiente en todas instancias organizacionales.

\section{REVISIÓN DE LITERATURA}

\section{El desempeño y el aprendizaje en las organizaciones}

Las organizaciones son concebidas como entes sociales cuya administración tiene como fin alcanzar objetivos colectivos bajo principios de cooperación (Arellano-Gault, 2014), para lo cual se necesita de personal competente. Estas son entidades que cumplen un rol social de suprema importancia y constituyen un poderoso instrumento de coordinación de personas y recursos. Cuando se trata del recurso humano, el tema se vuelve inmensamente complejo, debido a que se dan relaciones múltiples en varios sentidos, en donde, existen efectos de las personas sobre la organización y viceversa.

Hellriegel, Jackson, \& Slocum (2005) afirman que una planeación cuidadosa y un trabajo arduo contribuyen significativamente a una administración eficaz. Las organizaciones se desenvuelven bajo principios administrativos, dentro de ellos, la Administración Estratégica y Gestión del Talento Humano son dos aspectos importantes de la Administración que van de la mano en el desarrollo de las competencias de su gente y el cumplimiento de los objetivos organizacionales. La Administración Estratégica es el proceso de formular, implementar estrategias y evaluar los resultados obtenidos según decisiones tomadas en todas las áreas o departamentos de la empresa, para el cumplimiento de los objetivos organizacionales (David, 2007). La formulación de objetivos y estrategias se plantean para periodos generalmente largos, mientras que su monitoreo y evaluación se lleva a cabo de manera sistemática en periodos cortos, a fin de enrumbar las acciones hacia el propósito de la organización. 
Dentro de la Administración Estratégica los objetivos y estrategias formuladas, suelen tener cuatro perspectivas organizacionales: financiera, del cliente, interna, aprendizaje y crecimiento (Fernández, 2001), que se monitorean a través de indicadores. De estas perspectivas el aprendizaje es la más susceptible de caer en el campo de la subjetividad en razón de que no se establecen sus impactos en la organización, ni se analiza con frecuencia la relación entre la capacitación recibida y el mejoramiento del desempeño (Castañeda, 2002), pues es difícil establecer con claridad la incorporación de los nuevos conocimientos en el puesto de trabajo, los cuales prácticamente se diluyen en el tiempo.

Para una Administración Estratégica eficaz, se requiere de estrategas, los estrategas deben demostrar los resultados estratégicos del desempeño tanto del personal como de toda la organización en el largo plazo, dado que, por su naturaleza, no es factible observarlos a plazos cortos. Durante todo el proceso, se debe cuidar la alienación permanente al enfoque de mejora continua (Gutiérrez-Pulido, 2010), a fin de acortar brechas entre lo planificado y los logrado, tanto como sea posible, en cuanto, a recursos, como a tiempos establecidos. En toda organización el factor humano es el más importante (Alles, 2005), de hecho, no se concibe una organización sin la participación de personas. Como es conocido, los grupos humanos exhiben comportamientos variados que resultan de la intervención de múltiples variables endógenas y exógenas, provenientes tanto del entorno personal, como laboral; por lo tanto, es imperativo que las organizaciones propicien comportamientos satisfactorios de su personal, basados en el aprendizaje para mejorar el desempeño en la organización (Stable, 2012) que deben estar atados a la filosofía, valores, políticas y prácticas institucionales y visibles en su plan estratégico.

La importancia creciente de la Gestión del Talento Humano radica en que las competencias desarrolladas de los empleados de una organización no están disponibles para los competidores (Chiavenato 2007), pues, resulta sumamente difíciles de comprarlas, transferirlas o imitarlas. La Gestión del Talento Humano como una arista de la Administración Estratégica, contempla objetivos, estrategias, metas y recursos que son evaluados de modo permanente a fin de asegurar el desarrollo de la organización. Sin la respiración, no existe probabilidad de supervivencia humana, así mismo, sin evaluación, es imposible que las organizaciones tengan vida, de modo que, el desempeño debe ser evaluado, y la evaluación, debe ser diseñada apropiadamente para aspirar a la mejora continua. La evaluación del desempeño debe plantearse con un enfoque sistémico. Esta debe ser comprendida como un sistema para evidenciar de la mejor manera posible los encadenamientos causa-efecto de todos los componentes estratégicos de la organización, y debe ser construido con suficiente respaldo teórico (Arellano-Gault, 2014), y no solo ser adoptado, como una herramienta técnica de aplicación aislada, sostenida en su sofisticación.

El enfoque de sistemas es ampliamente congruente con las organizaciones, de tal manera que, una organización es concebida como un todo en la que sus componentes interactúan como una sola macro-unidad. De acuerdo a la teoría general de sistemas, ningún sistema puede ser entendido a través de sus elementos por separado. Un sistema implica la interacción e interdependencia de todas sus partes, en este caso, de modo integrado dentro de la organización. 
Un Sistema de Evaluación de Desempeño (SED), sirve como un gran mecanismo que evalúa a toda la organización. El diseño de un SED se articula con la planeación estratégica a fin de que sus objetivos, estrategias y recursos se orienten a un solo norte, y se sujeten a la misión y visión de la organización. El desempeño organizativo es motivo de estudio permanente por la gran cantidad de variables que influyen sobre él. Así, Arismendy-Morales y Santis-Aguas (2011), manifiestan que en las universidades el desempeño laboral es afectado por el clima organizacional y que no es lo mismo simplemente acudir a trabajar, que demostrar un eficiente desempeño en el trabajo. Por otro lado, Santamaría-Escobar y Pertuz-Martínez (2013) señalan que la clave del mejoramiento del desempeño empresarial es contar con una certificación de calidad, con ello a más de abrirse a una multitud de variables de gran de gran relevancia, se da paso de una manera más formal a las dimensiones del aprendizaje organizacional.

El enfoque de mejora continua en la evaluación del desempeño y su relación con el aprendizaje, tiene el respaldo de los modelos de excelencia de la calidad, especialmente en aquellos cuyos elementos que forman parte de la estructura del modelo, son precisamente el aprendizaje y la gestión del conocimiento, tal es el caso de los modelos Malcom Braldrige, European Foundation for Quality Management (EFQM) (Camisón, 2007), que exigen resultados concretos con evidencias claras. No es nuevo enunciar que no existe una receta para el diseño y aplicación de un SED, de existirlo, no sería más que una utopía que se traduciría en expectativas fallidas. Arellano-Gault, (2014) señala que un SED, es un mecanismo que intenta hacer más simple la compleja dinámica empresarial, que llega a ser un conjunto de aproximaciones a la realidad y constituye un instrumento de apoyo a la gestión. Un SED sirve de apoyo a la toma de decisiones y a la rendición de cuentas en las organizaciones, dado que aporta con parámetros establecidos a los que se recurre de modo indispensable, en mayor o menor magnitud, tanto en el quehacer cotidiano, como en el corto, mediano y largo plazo.

Una organización que quiere mejorar su desempeño debe revisar de manera sistemática y sostenida su estrategia (Arellano-Gault, 2014), pues como se sabe, los procesos estratégicos son procesos de análisis y síntesis, en donde el análisis concierne a la planeación y la síntesis en la aplicación de la estrategia (Mintzberg, Ahlstrand, Joseph, Henry Mintzberg, \& others, 1999), logrando la integración de ambas partes, en la toma de decisiones. Si bien, Bozeman (1987) manifiesta que "todas las organizaciones son públicas" dado que, existe algún grado de injerencia de lo público, aun en aquellas comúnmente conocidas como privadas; es necesario establecer que, para el diseño del SED, en organizaciones de diversa índole, se debe tomar en cuenta el marco normativo interno y externo, los procesos que ocurren internamente, y las instancias a las que se rinde cuentas. Tanto las organizaciones comúnmente llamadas públicas, como las privadas, deben desarrollar un SED acorde a la naturaleza de su organización y al contexto en el que se desenvuelven para aplicarlo con una actitud reflexiva en la búsqueda de mejores acercamientos a la realidad.

Es menester señalar que, la evaluación del desempeño debe estar dentro de una línea de pensamiento, que permita que se la entienda como una oportunidad de aprendizaje y mejoramiento continuo, y no como un simple cumplimiento de requisitos, de tal modo que, esté sujeta a continua observación, investigación y desarrollo. 
El proceso de diseño y aplicación de un SED responden a encadenamientos de aprendizajes (Arellano-Gault, 2014). Esto implica el establecimiento de claras relaciones causa-efecto, tomando en cuenta aquellas actividades que agregan valor tanto al cliente como a la organización. A lo anterior se añade que todo proceso debe ser llevado a cabo con empoderamiento, siempre que agregue valor a los clientes externos e internos. La naturaleza de la organización sin duda afecta al diseño y aplicación de un SED, cuando se trata de un SED para una institución educativa, existen varios elementos distintivos propios del servicio que ofrece.

En la construcción e implementación de un SED docente en Chile (Assaél \& Pavez, 2008) publicado en Revista Iberoamericana de Evaluación Educativa, se estableció que un SED debe ser de carácter formativo, tal que, permita desarrollar niveles altos de desempeño, funcionar de manera articulada, llevarse a cabo en diferentes direcciones partiendo de la autoevaluación, seguida de la hetero-evaluación y co-evaluación, contar con estándares para orientar a la toma de decisiones de acciones inmediatas, ser aplicado en forma gradual a fin de que todos los elementos del sistema se vayan acoplando a medida que este se fortalece, preparar las condiciones apropiadas tanto de personas, como de recursos y procesos para su efectiva aplicación, contar con el marco administrativo y legal que dé cobertura a su implementación al amparo del organismo gubernamental regulador en el campo de la educación.

Lo anterior induce a la utilización de herramientas de revisión de los SED, con las que se establezcan los parámetros de evaluación. Es importante que los parámetros y estándares sean socializados y comprendidos por todos para su aplicación efectiva. Una herramienta de revisión de un SED, debe provenir de un proceso de análisis y acuerdos con las entidades reguladoras, y debe constituirse en una herramienta de resolución de conflictos. Para su aplicación es menester que se definan claramente los procesos de evaluación, así como los indicadores que faculten el desarrollo humano, puesto que, el propósito principal de la evaluación del desempeño, al amparo de la Gestión del Talento Humano, es propiciar una gestión humana enriquecedora (Saldarriaga Ríos, 2008) dentro de cada actividad, desde todas las aristas posibles. Los procesos institucionales en el marco de la educación están sujetos a las políticas educativas dictaminadas por los organismos de gobierno, e influenciados por las políticas sociales y económicas vigentes en cada país. Para Assaél \& Pavez (2008) en la implementación de un sistema de evaluación en una institución educativa, la evaluación docente es un asunto polémico que se ve afectado por la definición de su rol, así como de las posiciones ideológicas, lo que se extiende también al personal administrativo y que debe ser permanentemente adaptado a su entorno (Santamaría-Escobar, 2013). Los resultados de una evaluación de desempeño faculta cotejar lo planificado con lo obtenido e induce a la toma de decisiones racionales (Simon, 2011), a fin de cerrar brechas, corregir errores, potencializar fortalezas, superar debilidades, mitigar amenazas y aprovechar oportunidades (David, 2007).

\section{Aprendizaje organizacional}

El aprendizaje organizacional es un proceso de mejora continua que involucra a individuos, grupos y organizaciones, que de una manera dinámica crea nuevas capacidades y juega un papel primordial en la productividad así como en otras variables que propician un impacto positivo en los resultados empresariales (Vargas-Hernández, 
Jiménez, Calderón, Casas \& Palomares, 2016). El aprendizaje es un eje transversal en las organizaciones, que, si bien ha sido generalmente abordado desde el ámbito educativo, la investigación, análisis y aplicación en las organizaciones data de alrededor de los años setenta con Schon, Argyris (1978) quienes han explicado cómo el conocimiento se genera en las organizaciones y ha tenido notables repercusiones entorno a su desarrollo.

Las dimensiones del aprendizaje organizacional son: la adquisición del conocimiento, distribución del conocimiento, memoria o registro de conocimiento y desaprendizaje (Santos Vijande, Sanzo Pérez, García Rodríguez, \& Trespalacios Gutiérrez, 2009). Cabe mencionar que la relevancia del desaprendizaje está explicada en virtud de que para su aplicación requiere de un importante nivel de consciencia y voluntad para tomar decisiones de cambio y rectificación de los errores cometidos en el trabajo. En las organizaciones, el aprendizaje se encuentra presente en toda actividad y tiene lugar en tres momentos: individual, de coordinación y de reflexión. Para la ejecución de tareas es necesario su coordinación, y cuando se las realizas, se reflexiona en alguna medida sobre la manera en que se la ha ejecutado en el puesto de trabajo, a fin de procurar mejorar el desempeño; entonces, se comprueba la naturaleza dinámica del aprendizaje en el entorno laboral, a partir del cual surge el nuevo conocimiento, en donde las relaciones de confianza desarrolladas, han reemplazado al liderazgo basado en el control y el miedo (Echeverría, 2000) .

A través del aprendizaje las organizaciones empiezan a generar conocimiento nuevo, este nuevo conocimiento suele empezar por una persona y se va transformando de tácito a explícito a medida que se lo comparte (Nonaka \& Takeuchi, 2000), con la imprescindible utilización, tanto del lenguaje figurativo y de simbolismos, como del lenguaje formal, de modo que se va posicionando a niveles grupales, organizacionales e interorganizacionales. La transferencia del conocimiento es una condición sine qua non dentro del aprendizaje y la gestión del conocimiento, así el conocimiento tácito propio de una persona y difícil de ser comunicado, pasa a conocimiento explícito de tipo formal que puede ser sistematizado y registrado en las memorias de la organización. La ventaja de que el flujo de conocimiento fluya de una manera efectiva en todas sus fases o dimensiones es que se encuentre disponible en la organización, pues es el resultado de haber sido transformado del plano individual, al colectivo y al organizacional, por lo que se debe buscar las maneras de propiciar su transformación eficiente.

El aprendizaje debe convertirse en un factor constante parte del estilo de vida en las organizaciones, que no busca solamente el desarrollo de competencias y capacidades de acción, sino de competencias que faculten el aprender a aprender. Para que los miembros de una organización logren el aprender a aprender de una manera continua y sostenida y conviertan a una organización común en una organización inteligente, se necesita el cultivo de varias disciplinas. Senge (2012), manifiesta que las cinco disciplinas que necesita desarrollar la organización inteligente son: el pensamiento sistémico, el dominio personal, los modelos mentales, la construcción de una visión compartida y el aprendizaje en equipo, con lo que la organización será capaz de crear y modificar su propia realidad. Mediante el aprendizaje en equipo las organizaciones crean sinergia y desarrollan una capacidad superior a la suma de las capacidades individuales de sus miembros (Senge, 2005). 
Para Kofman (2001), el aprendizaje se produce a diferentes niveles, el nivel más básico (cero) permite identificar errores, pero no induce a su corrección, sino al abandono de la tarea. Una vez que se haya detectado un error, en el nivel uno del aprendizaje, se corrige el error. Mientras que en el niel dos del aprendizaje, se provoca un cambio en el conjunto de acciones y objetivos como parte de una nueva concepción del problema. Finalmente, en el aprendizaje de nivel tres, se logra una nueva interpretación de las cosas en base al surgimiento de un nuevo paradigma. El nuevo paradigma da lugar a una organización de estado superior. A partir de la revisión de la literatura se determinó que el desempeño y el aprendizaje organizacional están relacionados (Zapata \& Ramírez, 2010), en base a lo cual se ha encontrado la suficiente motivación y respaldo teórico para emprender la presente investigación con el fin de contribuir al mejoramiento del desempeño de la facultad objeto de estudio, así pues Stable (2011) corrobora lo indicado y señala que la capacidad de aprendizaje de la organización contribuye a la mejora de su desempeño,

Con lo expresado, se establece la necesidad de expandir el pensamiento hacia nuevos horizontes, tomando en cuenta el análisis de relaciones causa-efecto, con una perspectiva dinámica y global, capaz de modificar los esquemas mentales vigentes (Senge, 2012). El propósito que se persigue es que las organizaciones potencien el desempeño en torno al aprendizaje y gestionen el conocimiento, de modo que, se conviertan en entes inteligentes generadores de riqueza. Las hipótesis planteadas en función del desempeño y las dimensiones del aprendizaje para el caso de la Facultad de Contabilidad y Auditoría de la Universidad Técnica de Ambato, son:

- Entre la adquisición del conocimiento y el desempeño organizacional existe una relación significativa positiva.

- Entre la distribución del conocimiento y el desempeño organizacional existe una relación significativa positiva.

- Entre la memoria organizacional y el desempeño organizacional existe una relación significativa positiva.

- Entre el desaprendizaje y el desempeño organizacional existe una relación significativa positiva.

- Entre el aprendizaje organizacional y el desempeño organizacional existe una relación significativa positiva.

\section{METODOLOGÍA}

Esta investigación es de tipo descriptivo correlacional, de corte transversal (Ynoub, 2015). El enfoque es cuali-cuantitativo, bajo el que se estableció la relación entre las variables, aprendizaje organizacional y desempeño. La Universidad Técnica de Ambato (UTA), fundada en 1962, es la institución pública de educación superior más importante de la provincia de Tungurahua, ubicada en la zona central de Ecuador, cuenta con aproximadamente 16000 estudiantes y 10 facultades entre las cuales la Facultad de Contabilidad y Auditoría (FCAUD) es la más antigua, pues fue con esta facultad con la que la universidad tuvo sus inicios. Esta facultad cuenta con 1753 estudiantes en las carreras de Contabilidad y Auditoría, Economía e Ingeniería Financiera. En esta investigación se utilizó a la modalidad documental y de campo, en razón de que, por una parte, se acudió a la revisión bibliográfica de los fundamentos teóricos e investigaciones previas reportadas en artículos científicos relacionados con las variables de estudio, y a la 
revisión de la información del plan estratégico y evaluación del desempeño de la FCAUD al que se aplicó una lista de chequeo para determinar la presencia de elementos del aprendizaje y del desempeño.

Por otra parte, se acudió a la investigación de campo para recopilar información en el lugar de los hechos. Para la recolección de los datos se utilizó una encuesta estructurada con 23 preguntas ( 5 de adquisición del conocimiento, 5 de distribución del conocimiento, 4 de memoria organizacional, 4 de desaprendizaje y 5 desempeño), las preguntas fueron adaptadas de los instrumentos utilizados en investigaciones de aprendizaje organizacional (Santos Vijande et al., 2009), y de medición del desempeño organizacional, en términos de percepción de desempeño (Zapata \& Ramírez, 2010). Las respuestas fueron expresadas en escala de Likert con equivalencia de 1 para totalmente en desacuerdo y 5 para totalmente de acuerdo. La encuesta se aplicó a una muestra de 63 docentes elegidos al azar de un universo de 75. La muestra fue calculada con la fórmula para población finita utilizando el 95\% de nivel de confianza y el 5\% de error.

El tratamiento estadístico de los datos se llevó a cabo con el programa SPSS, versión 19, con el que se determinaron los estadísticos descriptivos (medias y desviaciones típicas) de las respuestas correspondientes a las dimensiones del aprendizaje y del desempeño. Se calculó los coeficientes de correlación a fin de probar las hipótesis y establecer la relación entre las variables. El cuestionario de la encuesta se evaluó a través de la prueba de fiabilidad Alfa de Cronbach cuyo coeficiente indicó la consistencia interna del instrumento.

\section{RESULTADOS Y DISCUSIÓN}

Los resultados de la determinación del Alfa de Cronbach para las preguntas del cada una de las dimensiones del aprendizaje organizacional fueron: para adquisición del conocimiento, 0,750; para distribución del conocimiento, 0,736; para memoria organizacional, 0,891; para desaprendizaje, 0,814 y para desempeño 0,812. Estos valores revelaron que para cada dimensión el cuestionario es confiable; pues Gómez-Romero et al. (2015) al hacer el análisis estadístico en el mismo campo temático, manifiesta que valores entre 0,7 y 0,8 son considerados como respetables y entre 0,8 y 0,9 como muy buenos. Por otro lado, el análisis la fiabilidad del instrumento total arrojó un valor del de 0,918 que indica que de manera general que el instrumento utilizado en la presente investigación es fiable. En la siguiente tabla se resumen los estadísticos descriptivos de las dimensiones del aprendizaje organizacional y el desempeñó en donde, al analizar los promedios se encuentra que los valores más altos están ubicados en 3,77 y 3,76 y corresponden al desaprendizaje y a la memoria organizativa respectivamente, mientras que se percibe un ligero descenso en la adquisición y distribución del conocimiento.

Tabla 1: Estadísticos descriptivos de las dimensiones del aprendizaje organizacional y desempeño

\begin{tabular}{cccccc}
\hline & $\begin{array}{c}\text { Adquisición } \\
\text { conocimiento }\end{array}$ & $\begin{array}{c}\text { Distribución } \\
\text { conocimiento }\end{array}$ & $\begin{array}{c}\text { Memoria } \\
\text { organizativa }\end{array}$ & Desaprendizaje & Desempeño \\
\hline Media & 3,56 & 3,69 & 3,76 & 3,77 & 4,26 \\
Desv. típica & 0,758 & 0,731 & 0,897 & 0,847 & 0,686 \\
Coef. variación & $21,3 \%$ & $19,8 \%$ & $23,9 \%$ & $22,5 \%$ & $16,1 \%$ \\
\hline
\end{tabular}

Fuente: Datos de las encuestas procesados en SPSS. 
Los resultados del desaprendizaje, dentro de la escala de Likert están muy cercanos a la categoría de acuerdo, con lo que se determina que los docentes están de acuerdo en cambiar su manera de hacer las cosas a fin de adaptarse a las nuevas situaciones del entorno educativo y que las experiencias fallidas les sirve para aprender. También están de acuerdo en el cuestionamiento permanente a su forma de actuar sin que existan creencias preestablecidas e inamovibles en el área de su desempeño. Los resultados de la dimensión de la memoria organizativa señalan un acuerdo general de parte del personal docente en que la FCAUD conserva bases de datos, ficheros, o archivos de la experiencia de tal forma que pueden utilizarlos con facilidad, además afirman que se dispone de medios para conservar la información adquirida a través de la experiencia, aunque exista rotación en los empleados o docentes. Concuerdan también en que la información a la que tiene acceso el personal docente, es información actualizada.

Los resultados de la adquisición y distribución del conocimiento exhiben un ligero descenso con relación a las dos dimensiones anteriormente descritas, sin embargo, también se los ubica en la categoría de acuerdo. A partir de lo indicado y en base al contenido del cuestionario aplicado, se manifiesta que el grupo investigado afirma que la FCAUD obtiene información para la mejora continua del currículo mediante acercamientos con los sectores productivos, empresariales y estudiantiles. También obtiene información del personal administrativo y docente para la mejorar del servicio educativo, hace evaluaciones de sus competidores y adopta aquellas prácticas y técnicas que considera útiles. El conocimiento nuevo proveniente de todas estas fuentes es compartido en reuniones periódicas, así como dentro de los equipos de trabajo y en las distintas comisiones.

La variable desempeño reportó el valor más alto, con un promedio 4,26 con lo que se determinó que los docentes, tienen una alta percepción del desempeño organizacional, consideran a la FACUD como exitosa, e indican que alcanzan los niveles de desempeño deseados tanto cuando trabajan en equipo como cuando lo hacen individualmente por lo que se consideran satisfechos de trabajar en la facultad. Finalmente, están de acuerdo en que la FCAUD satisfice las necesidades de los estudiantes al prepararlos de acuerdo al entorno empresarial. Mediante el coeficiente de variación se estableció que las respuestas más homogéneas se encuentran en el desempeño, con un 16\% de variación, mientras que es en la memoria organizativa donde existe la mayor variación en las respuestas con respecto a la media (23,9\%). Esto que los criterios tienen un mayor consenso en los criterios referentes al desempeño.

El análisis llevado a cabo en el estudio de correlación (Spearman) indicó que existe evidencia estadística significativa de la correlación directa entre la percepción del desempeño y el aprendizaje organizacional en la FCAUD ( $r=0,873)$, para un nivel de significancia del 5\%, correlación que es considerada como muy buena (Anderson, 2011). En la tabla 2 se reportan los coeficientes de correlación de Spearman que reportan la correlación entre cada una de las dimensiones del aprendizaje organizacional y de éstas con el desempeño. 
Tabla 2. Correlaciones (Spearman) entre las dimensiones del Aprendizaje organizacional y el desempeño

\begin{tabular}{|c|c|c|c|c|c|c|}
\hline & \multicolumn{2}{|c|}{$\begin{array}{l}\text { Adquisición } \\
\text { conocimiento }\end{array}$} & $\begin{array}{l}\text { Distribución } \\
\text { conocimiento }\end{array}$ & Desempeño & $\begin{array}{c}\text { Memoria } \\
\text { organizacional }\end{array}$ & Desaprendizaje \\
\hline $\begin{array}{l}\text { Adquisición } \\
\text { conocimiento }\end{array}$ & Sig. & 1 & & & & \\
\hline \multirow{2}{*}{$\begin{array}{l}\text { Distribución } \\
\text { conocimiento }\end{array}$} & & $0,614^{\star \star}$ & 1 & & & \\
\hline & Sig. & 0,000 & & & & \\
\hline \multirow{2}{*}{ Desempeño } & & $0,473^{* *}$ & $0,614^{\star \star}$ & 1 & & \\
\hline & Sig. & 0,000 & 0,000 & & & \\
\hline \multirow{2}{*}{$\begin{array}{c}\text { Memoria } \\
\text { organizativa }\end{array}$} & & $0,312^{*}$ & $0,558^{\star *}$ & $0,711^{\star \star}$ & 1 & \\
\hline & Sig. & 0,013 & 0,000 & 0,000 & & \\
\hline \multirow{2}{*}{ Desaprendizaje } & & $0,402^{* *}$ & $0,591^{\star \star}$ & $0,483^{* *}$ & $0,534^{\star *}$ & 1 \\
\hline & Sig. & 0,001 & 0,000 & 0,000 & 0,000 & \\
\hline
\end{tabular}

Fuente: Elaboración de los autores con base a los resultados de las encuestas

Se encontró una buena correlación entre la adquisición y la distribución del conocimiento $(0,614)$, mientras que la correlación entre la adquisición del conocimiento y la memoria organizativa, así como con el desaprendizaje, (0,312 y 0,402 respectivamente) es moderada. La correlación mejora un poco para la distribución del conocimiento y la memoria organizativa $(0,058)$, así como de ésta con el desaprendizaje $(0,591)$, pero sigue siendo moderada. De modo parecido sucede con la memoria organizativa y el desaprendizaje $(0,538)$. Por otro lado, se aprecia una buena correlación entre el desempeñó y la memoria organizativa $(0,711)$, así como con la distribución del conocimiento $(0,614)$, sin embargo, la correlación entre el desempeñó y la adquisición del conocimiento $(0,473)$ y el desaprendizaje $(0,483)$ es moderada.

Las asociaciones encontradas en todos los casos, mostraron el sentido positivo de cada relación. Adicionalmente, se demostró que el $76,2 \%$ del desempeño organizacional fue explicado en conjunto, por las dimensiones del aprendizaje organizacional (coeficiente de determinación $r^{\wedge}=0,762$ ). El sentido y la magnitud de los valores de los coeficientes hallados en la presente investigación guardan concordancia con lo reportado por Quispe \& Vigo (2017) quienes determinaron la interacción del aprendizaje organizacional y el desempeño laboral de los educadores de las instituciones educativas públicas de Perú.

Al aplicar las listas de chéquelo en todos los ejes del plan estratégico de la FCAUD, no se encontró la inclusión de elementos del aprendizaje, además de la ausencia de este en los procesos de evaluación de desempeño. Se determinó también que el sistema de evaluación vigente es un sistema impuesto por el organismo de gobierno, lo que se hace visible en su estructura y en los aspectos contemplados para darles cumplimiento a los que deben acogerse sin la opción de diálogos previos ni posteriores para ello.

Los resultados de la comprobación de hipótesis se muestran a continuación:

- La adquisición del conocimiento y el desempeño organizacional de la Facultad de Contabilidad y Auditoría tienen una correlación significativa moderada y positiva $(0,473)$. 
- La distribución del conocimiento y el desempeño organizacional de la Facultad de Contabilidad y Auditoría tienen buena correlación significativa y positiva $(0,614)$.

- La memoria organizacional y el desempeño organizacional de la Facultad de Contabilidad y Auditoría tienen una buena correlación significativa y positiva $(0,711)$.

- El desaprendizaje y el desempeño organizacional de la Facultad de Contabilidad y Auditoría tienen una relación significativa moderada y positiva $(0,483)$.

- El aprendizaje organizacional y el desempeño organizacional de la Facultad de Contabilidad y Auditoría revelan la presencia de una muy buena correlación significativa y positiva $(0,873)$.

Los resultados presentados en esta investigación permiten la identificación y posterior implementación de buenas prácticas de aprendizaje organizacional para potenciar el desempeño tanto de manera global de la unidad academica, como del talento humano que articuladamente brinda su importante contingente en el día a día, en virtud de que el aprendizaje organizacional favorece a un modelo de gestión participativo que amplía los campos de acción de todos los miembros de la organización Mayorca-Capataz \& Mayorca-Beltrán (2010).

\section{CONCLUSIONES}

En virtud se estableció que el aprendizaje organizacional está correlacionado positivamente con el desempeño, es recomendable incluirle en diseño del plan estratégico para poder monitorearlo y determinar su nivel de impacto en resultados de la gestión de la FCAUD, dado que al ser el aprendizaje organizacional una variable de perdurabilidad tiene un impacto positivo muy grande en el cambio y la productividad de las organizaciones (Vargas, et. al., 2016). Para el diseño de un SED, se aconseja la revisión de otros sistemas de este tipo, cuyo funcionamiento haya sido probado, en el que se analicen sus características y factores críticos de éxito, a fin de acoplarlos de modo integral, con criterios técnicos y éticos, a los propósitos institucionales, con un espíritu potenciador del talento humano en el que el aprendizaje sea un inductor efectivo del desempeño; de lo contrario, como lo manifiesta Pardo-Ramos (2006) en su investigación relativa al aprendizaje organizacional en entidades públicas, la fuerza laboral puede llegar a ser poco productiva, desmotivada y con conocimientos desactualizados, lo que se traduciría en una baja calidad del servicio educativo.

El sistema en mención contaría con indicadores y procesos establecidos, instituidos con un enfoque sistémico de mejora continua y de adaptación permanente con su entorno, pues, al amparo de la Teoría Situacional, el desempeño de las organizaciones depende en gran medida de su capacidad de adaptación al entorno (Santamaría-Escobar, \& Pertuz-Martínez, 2013). Es necesario que se impulse el desarrollo efectivo del aprendizaje en cada una de sus dimensiones, en razón de que, en todas ellas, se encontró evidencia estadística significativa de su interacción con el desempeño organizativo, tomando en cuenta aspectos tanto de lo individual, como de lo colectivo, que estuvieron comprendidos en algunas de las dimensiones del aprendizaje (adquisición, distribución y desaprendizaje).

Es necesario reflexionar en la manera en que se hacen las cosas en cuanto al manejo del conocimiento se refiere, e inducir a que las prácticas de la adquisición, distribución, 
memoria organizativa (registros) y desaprendizaje de conocimientos, cuenten con una alta dosis de intencionalidad, es decir, se recomienda que sean diseñadas dentro de un sistema de aprendizaje, y no ocurran de modo simple y aislado. Para ello, se sugiere además que se dé cumplimiento al requisito de que cada persona haya vivido previamente un proceso de sensibilización y esté consciente de lo que aprende y de cómo aprende, a fin de que puedan influir positivamente en un buen desempeño. De acuerdo con los resultados obtenidos, se recomienda tomar acciones inmediatas en la implementación de prácticas para la adquisición y distribución del conocimiento (correlación de 0,614) y que se instituyan prácticas de memoria organizativa (correlación de 0,711 ) en virtud del impulso que dará a la gestión del conocimiento en la facultad en conjunción con los factores adyacentes propios de la institución.

Por otro lado, se recomienda que se mire de cerca a los factores externos asociados que afectan al clima organizacional, por cuanto, éste afecta al desempeño tanto de los docentes como de los administrativos, como lo manifiestan Arismendy-Morales \& SantisAguas, (2011), en su estudio llevado a cabo en universidades del departamento de la Guajira, pues el buen desempeño es resultante de la planificación y aunque implique un proceso de ensayo y error, y no consiste en la simple y mecánica aplicación de herramientas de evaluación. De hecho, el ser humano no puede aprender ni desempeñarse de manera satisfactoria, en un ambiente deteriorado. Además, de acuerdo a lo establecido en los fundamentos teóricos, es necesario que el SED sea desarrollado de manera colaborativa con la participación de importantes stakeholders, tomando en cuenta de modo global los procesos de rendición de cuentas, tanto a los organismos rectores, como a la sociedad. Se recomienda que el sistema sea revisado periódicamente, en atención no solo al cumplimiento y comparación de los resultados obtenidos versus los reales, sino a los cambios introducidos en el plan estratégico, en función de las nuevas condiciones políticas y disposiciones del sistema educativo.

Sería recomendable que el CEAACES, y las IES tengan acercamientos encaminados al diálogo, para trabajar juntos en una reformulación del sistema de evaluación que potencie la academia, investigación, vinculación y la gestión, a fin de impulsar a la consecución de resultados concretos en beneficio de la sociedad, de modo que, el trabajo no se centre en la generación y recolección de evidencias, sino que esas evidencias se produzcan de manera natural, como el resultado del genuino cumplimiento de los objetivos de la misión educativa.

En torno a la investigación del aprendizaje organizacional se plantean varias preguntas, por ejemplo: ¿cómo las organizaciones deben promover y desarrollar el aprendizaje? ¿Cómo el aprendizaje se ve reflejado en el desempeño tanto del personal, como de la organización? ¿Cómo se puede monitorear y medir el aprendizaje a través del desempeño? Estas preguntas ameritan ser respondidas a través de la investigación en las organizaciones, tomando en cuenta factores específicos de influencia, como las diferencias en la estructura organizacional, procesos de aprendizaje propios de la naturaleza de cada organización, el tipo de organización sean de gobierno o privadas y otros de importancia que se irían enunciando a medida que se profundicen las investigaciones en torno a esta temática. 
Finalmente, se concluye que es relevante continuar investigando el aprendizaje organizacional en la Facultad de Contabilidad y Auditoría, que como es conocido en el medio, su desenvolvimiento es un referente para la institución. Esta investigación es aplicable en otras dependencias e instituciones, pero se recomienda que se aborden nuevas perspectivas relacionadas con aspectos particulares propios del desempeño del personal docente y administrativo a con miras a la implementación de buenas prácticas de aprendizaje organizacional.

\section{REFERENCIAS}

Alles, M. (2005). Desempeño por competencias para una evaluación de 360. Buenos Aires: Granica

Anderson, D., Sweeney,D., Williams, T. (2011). Estadística para Administración y Economía. México: McGrawHill

Arismendy-Morales M.y Santis-Aguas, E. (2011). Clima organizacional y desempeño laboral, una reflexión cuantitativa en las universidades del departamento de la Guajira. Revista Panorama Económico, 19, 163-176

Argyris, C., y Schon, D. (1978). Organizational learning: A theory of action perspective. REIS, 77, 345348.

Aramburu, N. (2000). Un estudio del aprendizaje organizativo desde la perspectiva del cambio. Universidad de Deusto. San Sebastián, Deusto. San Sebastián.

Arellano,D. (2014). Sistemas de Evaluación del Desempeño para organizaciones públicas. CIDE.

Assaél, J., y Pavez, J. (2008). La construcción e implementación del sistema de evaluación del desempeño docente chileno: principales tensiones y desafíos. Revista Iberoamericana de Evaluación Educativa. Recuperado de https://repositorio.uam.es/handle/10486/661518

Castañeda, D. I., y Ríos, M. F. (2007). Validación de una escala de niveles y condiciones de aprendizaje organizacional. Universitas Psychologica, 6(2), 245-254.

Camisón, C. (2007). Gestión de la Calidad: conceptos, enfoques, modelos y sistemas. Madrid: Pearson Education

Consejo de Evaluación, Acreditación y Aseguramiento de la Calidad de la Educación Superior. (2015). Modelo de Evaluación Institucional de Universidades y Escuelas Politécnicas. Recuperado de http://www.ceaaces.gob.ec/sitio/wp-content/uploads/2016/06/Modelo-deevaluacio\%CC\%81n-institucional-2016.pdf

Chiavenato, I. (2007). Administración de recursos humanos: el capital humano de las organizaciones. McGraw-Hill/Interamericana.

David, F. R. (2007). Conceptos de administración estratégica. México: Pearson.

Del Río Cortina, J., y Rojas, D. F. S. (2011). Perspectivas del aprendizaje organizacional como catalizador de escenarios competitivos/organizational learning perspectives as catalizers of competitive scenarios. Revista Ciencias Estratégicas, 19(26), 247.

Echeverría, R. (2000). La empresa emergente, la confianza y los desafíos de la transformación. Ediciones Granica S.A.

Fernández, A. (2001). El Balanced Scorecard. Revista de Antiguos Alumnos, 31-42.

Gómez-Romero, J., Villareal, D., y Villareal, F. (2015). Las incapacidades de aprendizaje organizacional y su relación con los estilos de aprendizaje organizacional en la Mipyme. Contaduría y administración, 60(3), 686-702. Recuperado de https://doi.org/10.1016/i.cya.2013.11.001

Gutiérrez-Pulido, H. (2010). Calidad total y productividad. McGraw Hill.

Hellriegel, D., Jackson, S. E., y Slocum, J. W. (2005). Administración: un enfoque basado en competencias. Thomson Learning. 
Kofman, F. (2001). Metamanagement: la nueva con-ciencia de los negocios: cómo hacer de su vida profesional una obra de arte. Grancia, Ediciones, S.A.

Mayorca-Capataz, E., y Mayorca-Beltrán, D. (2010). El aprendizaje organizacional una estrategia de cambio en la administración de hoy. Revista Panorama Económico, 18, 203-213

Nonaka, I. y Takeuchi, H. (2000). La empresa creadora de conocimiento. Gestión del conocimiento, $1-9$

Pardo-Ramos, O. (2006). El aprendizaje organizacional en entidades públicas del orden nacional del Departamento de Bolívar: Propuesta de mejoramiento. Revista Panorama Económico, 14, 882

Quispe, E., y Vigo, S. (2017). La interacción del aprendizaje organizacional y el desempeño laboral de los educadores de las instituciones educativas públicas. Revista de Investigación en Psicología, 20(2).

Saldarriaga Ríos, J. G. (2008). Gestión humana: tendencias y perspectivas. Estudios Gerenciales, 24(107), 137-159. Recuperado de https://doi.org/10.1016/50123-5923(08)70040-8

Sanabria, P., Telch, F., Rodas, A., Astudillo, M., y Estrada, S. (2015). Lineamientos básicos para una política de gestión del recurso humano para el contexto del sector público colombiano. Recuperado de https://papers.ssrn.com/sol3/papers. cfm?abstract id=2926970

Santamaría-Escobar, A.,y Pertuz-Martínez, A. (2013) Certificación de la Calidad ISO 9001: Clave del mejoramiento del desempeño empresarial. Revista Panorama Económico, 21, 191-213

Santos Vijande, M., Sanzo Pérez, M., García Rodríguez, N., y Trespalacios Gutiérrez, J. A. (2009). Procesos de aprendizaje en las pymes industriales españolas: efectos en la innovación, calidad de la oferta y resultados empresariales. INNOVAR Revista de Ciencias Administrativas y Sociales, 19(33). Recuperado de http://www.redalyc.org/htm//818/81819022004/

Senge, P. M. (2005). La Quinta Disciplina En La Practica. Ediciones Granica S.A.

Senge, P. M. (2012). La quinta disciplina: Como impulsar el aprendizaje en la organización inteligente. Ediciones Granica S.A.

Simon, H. A. (2011). El comportamiento administrativo: un estudio de los procesos de decisión en las organizaciones administrativas. Argentina: Errepar.

Stable, Y. (2011). Modelo de aprendizaje organizacional para organizaciones de información. Revista Cubana de Información en Ciencias de la Salud, 22(3). 237-250.

Stable, Y. (2012). Modelo y metodología de aprendizaje organizacional para el mejor desempeño de una organización de ciencia e innovación. Recuperado de https://www.researchgate.net/publication/280530689 modelo y metodologia de aprendizaje organizacional_para_el_mejor_desempeno_de_una_organizacion_de_ciencia_e_innovacion

Ynoub, R. (2015). Cuestión del Método. Aportes para una metodología crítica. (1a Edición). México, DF.: Cengage Learning Editores.

Vargas, J., Almanza, R., Calderón, P., Casas, R., y Palomares, F. (2016). Performance y aprendizaje organizacional bajo el enfoque de las teorías organizacionales. Perspectiva Socioeconómica.

Zapata, J. P., y Ramírez, J. A. (2010). Medición y validación del desempeño organizacional como resultado de acciones de aprendizaje. Revista Ciencias Estratégicas, 17(22), 251-271. 